$2007 / 05 / 25$

\title{
SIGNED WORDS AND PERMUTATIONS, V; A SEXTUPLE DISTRIBUTION
}

\author{
Dominique Foata and Guo-Niu Han
}

\begin{abstract}
We calculate the distribution of the sextuple statistic over the hyperoctahedral group $B_{n}$ that involves the flag-excedance and flag-descent numbers "fexc" and "fdes," the flag-major index "fmaj," the positive and negative fixed point numbers "fix + " and "fix ${ }^{-"}$ and the negative letter number "neg." Several specializations are considered. In particular, the joint distribution for the pair (fexc, fdes) is explicitly derived.
\end{abstract}

\section{Introduction}

As has been shown in our series of four papers ([FoHa07], [FoHa05], [FoHa06], [FoHa07a]), the length function " $\ell$ " (see [Bo68], p. 7, or [Hu90], p. 12) and the flag major index "fmaj" introduced by Adin and Roichman [AR01] have become the true $q$-analog makers for the calculation of various multivariable distributions on the hyperoctahedral group $B_{n}$ of the signed permutations. The elements of $B_{n}$ may be viewed as words $w=x_{1} x_{2} \cdots x_{n}$, where each $x_{i}$ belongs to the set $\{-n, \ldots,-1,1, \ldots, n\}$ and $\left|x_{1}\right|\left|x_{2}\right| \cdots\left|x_{n}\right|$ is a permutation of $12 \ldots n$. The set (resp. the number) of negative letters among the $x_{i}$ 's is denoted by Neg $w$ (resp. neg $w$ ). A positive fixed point of the signed permutation $w=x_{1} x_{2} \cdots x_{n}$ is a (positive) integer $i$ such that $x_{i}=i$. It is convenient to write $\bar{i}:=-i$ for each integer $i$. If $x_{i}=\bar{i}$ with $i$ positive, we say that $\bar{i}$ is a negative fixed point of $w$. The set of all positive (resp. negative) fixed points of $w$ is denoted by $\mathrm{Fix}^{+} w\left(\right.$ resp. Fix $\left.{ }^{-} w\right)$. Notice that $\mathrm{Fix}^{-} w \subset \mathrm{Neg} w$. Also let

$$
\operatorname{fix}^{+} w:=\# \mathrm{Fix}^{+} w ; \quad \operatorname{fix}^{-} w:=\# \mathrm{Fix}^{-} w .
$$

There are $2^{n} n$ ! signed permutations of order $n$. The symmetric group $\mathfrak{S}_{n}$ may be considered as the subset of all $w$ from $B_{n}$ such that Neg $w=\emptyset$. When $w$ is an (ordinary) permutation from $\mathfrak{S}_{n}$, then $\operatorname{Fix}^{-} w=\emptyset$, so that we define $\operatorname{Fix} w:=\operatorname{Fix}^{+} w$ and fix $w=\# \operatorname{Fix} w$.

2000 Mathematics Subject Classification. Primary 05A15, 05A30, 05E15, 33D15.

Key words and phrases. Hyperoctahedral group, length function, flag-major index, flag-excedance number, flag-descent number, signed permutations, fixed points, Lyndon factorization, decreases, even decreases, $q$-series telescoping. 


\section{DOMINIQUE FOATA AND GUO-NIU HAN}

Now, for each statement $A$ let $\chi(A)=1$ or 0 depending on whether $A$ is true or not. Besides the integer-valued statistics "fix ${ }^{+}, "$ "fix ${ }^{-}$" and "neg" the now classical flag-descent number "fdes", flag-major index "fmaj" and flag-excedance number "fexc" are also needed in the following study. They are defined for each signed permutation $w=x_{1} x_{2} \cdots x_{n}$ by

$$
\begin{aligned}
\operatorname{fdes} w & :=2 \operatorname{des} w+\chi\left(x_{1}<0\right) ; \\
\operatorname{fmaj} w & :=2 \operatorname{maj} w+\operatorname{neg} w ; \\
\operatorname{fexc} w & :=2 \operatorname{exc} w+\operatorname{neg} w ;
\end{aligned}
$$

where "des" is the number of descents des $w:=\sum_{i=1}^{n-1} \chi\left(x_{i}>x_{i+1}\right)$, "maj" the major index maj $w:=\sum_{i=1}^{n-1} i \chi\left(x_{i}>x_{i+1}\right)$ and "exc" the number of excedances exc $w:=\sum_{i=1}^{n-1} \chi\left(x_{i}>i\right)$.

Our intention is to calculate the distribution of the sextuple statistic (fexc, fdes, fmaj, fix ${ }^{+}$, fix ${ }^{-}$, neg) on the group $B_{n}$. This means that for each $n \geq 0$ the generating polynomial

$$
B_{n}\left(s, t, q, Y_{0}, Y_{1}, Z\right):=\sum_{w \in B_{n}} s^{\mathrm{fexc} w} t^{\mathrm{fdes} w} q^{\mathrm{fmaj} w} Y_{0}^{\mathrm{fix}{ }^{+} w} Y_{1}^{\mathrm{fix}^{-} w} Z^{\mathrm{neg} w}
$$

can be considered and, with a suitable normalization, the generating function for those polynomials be summed. Furthermore, the summation is to yield an appropriate closed form. Finally, the calculation must be compatible with the symmetric group $\mathfrak{S}_{n}$ in the sense that when the variable $Z$ is given the zero value, earlier results derived for that group are to be recovered. As is shown below, this goal will be achieved by working in the algebra of $q$-series.

Our derivation has been motivated by the following recent statistical studies on $B_{n}$ and $\mathfrak{S}_{n}$. In [FoHa07a] and [FoHa07b] we have respectively calculated the generating functions for the polynomials $B_{n}\left(1, t, q, Y_{0}, Y_{1}, Z\right)$ and $A_{n}\left(s, t, q, Y_{0}\right)$, where

$$
A_{n}(s, t, q, Y):=\sum_{\sigma \in \mathfrak{S}_{n}} s^{\operatorname{exc} \sigma} t^{\operatorname{des} \sigma} q^{\operatorname{maj} \sigma} Y^{\mathrm{fix} \sigma}
$$

and shown that by giving certain variables specific values the former statistical results on $\mathfrak{S}_{n}$ and $B_{n}$ could be reobtained. Note that, using the previous definitions of "fexc," "fdes" and "fmaj," the latter polynomial is nothing but $B_{n}\left(s^{1 / 2}, t^{1 / 2}, q^{1 / 2}, Y, 0,0\right)$.

In the diagram of Fig. 1 the polynomials on the top (resp. bottom) level are specializations of the polynomial $B_{n}\left(s, t, q, Y_{0}, Y_{1}, Z\right)$ (resp. $\left.A_{n}(s, t, q, Y)\right)$. Each vertical arrow is given a $(Z=0)$-label. This means that when $Z$ is given the 0 -value, each polynomial $B_{n}(\cdots)$ is transformed into the corresponding polynomial $A_{n}(\cdots)$. The other arrows labelled $s$, $t$ and $Z$ indicate that each target polynomial is mapped onto the source polynomial when $s$ (resp. $t$, resp. $Z$ ) is given the 1 -value. 


\section{A SEXTUPLE DISTRIBUTION}

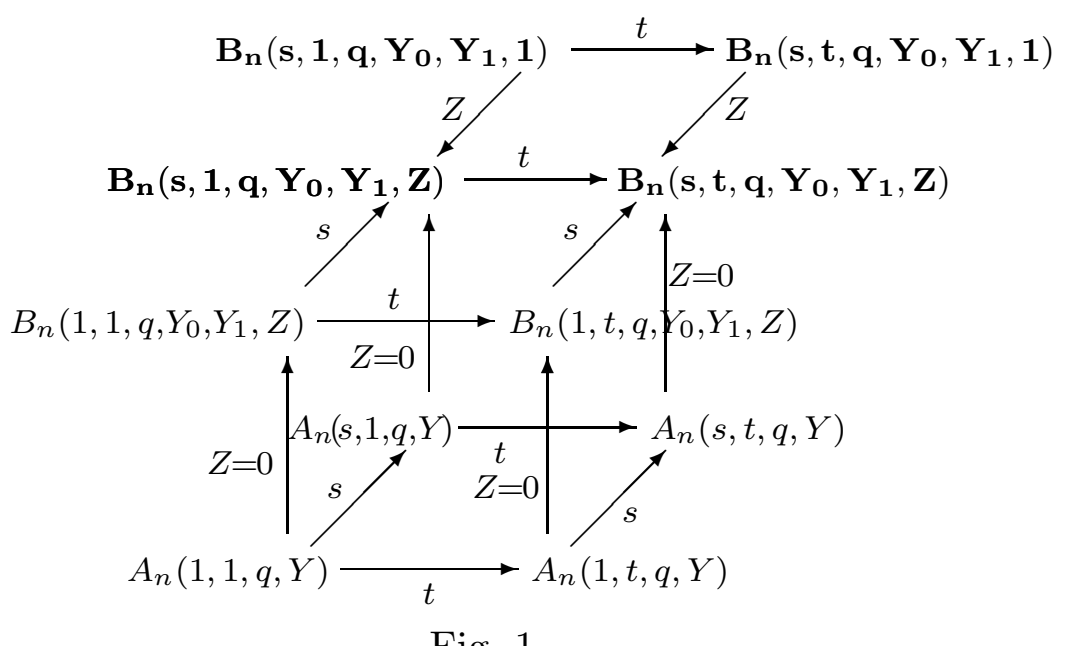

Fig. 1

The polynomials appearing on the top are all generating polynomials for the group $B_{n}$. Four of them are reproduced in boldface. Their factorial generating polynomials are derived in the present paper. The generating functions for the other six polynomials have been explicitly determined in earlier papers: $A_{n}(1,1, q, Y)$ and $A_{n}(1, t, q, Y)$ by Gessel and Reutenauer [GeRe03], then $A_{n}(s, 1, q, Y)$ by Shareshian and Wachs [ShWa06], furthermore $A_{n}(s, t, q, Y)$ in [FoHa07b], finally $B_{n}\left(1,1, q, Y_{0}, Y_{1}, Z\right)$ and $B_{n}\left(1, t, q, Y_{0}, Y_{1}, Z\right)$ in [FoHa07a].

The classical notation on $q$-series will be used. First, the $q$-ascending factorials

$$
\begin{gathered}
(a ; q)_{n}:= \begin{cases}1, & \text { if } n=0 \\
(1-a)(1-a q) \cdots\left(1-a q^{n-1}\right), & \text { if } n \geq 1 .\end{cases} \\
(a ; q)_{\infty}:=\prod_{n \geq 1}\left(1-a q^{n-1}\right) ;
\end{gathered}
$$

then, the two $q$-exponentials (see [GaRa90, chap. 1])

$$
e_{q}(u)=\sum_{n \geq 0} \frac{u^{n}}{(q ; q)_{n}}=\frac{1}{(u ; q)_{\infty}} ; \quad E_{q}(u)=\sum_{n \geq 0} q^{\left(\begin{array}{c}
n \\
2
\end{array}\right)} \frac{u^{n}}{(q ; q)_{n}}=(-u ; q)_{\infty} .
$$

The main purpose of this paper is to prove the following theorem.

Theorem 1.1. For each $n \geq 0$ let $B_{n}\left(s, t, q, Y_{0}, Y_{1}, Z\right)$ be the generating polynomial for the hyperoctahedral group $B_{n}$ by the six-variable statistic (fexc, fdes, fmaj, fix ${ }^{+}, \mathrm{fix}^{-}$, neg) as defined in (1.2). Then, the factorial generating function for the polynomials $B_{n}\left(s, t, q, Y_{0}, Y_{1}, Z\right)$ is given by:

$$
\begin{aligned}
& \sum_{n \geq 0}(1+t) B_{n}\left(s, t, q, Y_{0}, Y_{1}, Z\right) \frac{u^{n}}{\left(t^{2} ; q^{2}\right)_{n+1}} \\
& \quad=\sum_{r \geq 0} t^{r} \frac{\left(u ; q^{2}\right)\lfloor r / 2\rfloor+1}{\left(u Y_{0} ; q^{2}\right)_{\lfloor r / 2\rfloor+1}} \frac{\left(-u s q Y_{1} Z ; q^{2}\right)_{\lfloor(r+1) / 2\rfloor}}{\left(-u s q Z ; q^{2}\right)_{\lfloor(r+1) / 2\rfloor}} F_{r}(u ; s, q, Z),
\end{aligned}
$$


where

$$
\begin{aligned}
& F_{r}(u ; s, q, Z) \\
& =\frac{\left(u s^{2} q^{2} ; q^{2}\right)_{\lfloor r / 2\rfloor}\left(1-s^{2} q^{2}\right)\left(u ; q^{2}\right)_{\lfloor(r+1) / 2\rfloor}\left(u ; q^{2}\right)_{\lfloor r / 2\rfloor}}{\left(u ; q^{2}\right)_{\lfloor r / 2\rfloor+1}\left(\left(u ; q^{2}\right)_{\lfloor(r+1) / 2\rfloor}\left(\left(u ; q^{2}\right)_{\lfloor r / 2\rfloor}-s^{2} q^{2}\left(u s^{2} q^{2} ; q^{2}\right)_{\lfloor r / 2\rfloor}\right)\right.} \\
& \left.+s q Z\left(u ; q^{2}\right)_{\lfloor r / 2\rfloor}\left(\left(u ; q^{2}\right)_{\lfloor(r+1) / 2\rfloor}-\left(u s^{2} q^{2} ; q^{2}\right)_{\lfloor(r+1) / 2\rfloor}\right)\right) .
\end{aligned}
$$

The factorial generating functions for the other polynomials written in boldface in the diagram of Fig. 1 are shown to be specializations of the factorial generating function for the six-variable polynomials $B_{n}\left(s, t, q, Y_{0}, Y_{1}, Z\right)$, as stated in the next three Corollaries.

Corollary 1.2. The factorial generating function for the polynomials $B_{n}\left(s, 1, q, Y_{0}, Y_{1}, Z\right)$ is given by

$$
\begin{aligned}
\sum_{n \geq 0} B_{n}(s & \left., 1, q, Y_{0}, Y_{1}, Z\right) \frac{u^{n}}{\left(q^{2} ; q^{2}\right)_{n}}=\frac{e_{q^{2}}\left(u Y_{0}\right) E_{q^{2}}\left(u s q Y_{1} Z\right)}{E_{q^{2}}(u s q Z)} \\
& \times \frac{\left(1-s^{2} q^{2}\right)}{e_{q^{2}}\left(u s^{2} q^{2}\right)-s^{2} q^{2} e_{q^{2}}(u)+s q Z\left(e_{q^{2}}\left(u s^{2} q^{2}\right)-e_{q^{2}}(u)\right)} .
\end{aligned}
$$

Corollary 1.3. The factorial generating function for the polynomials $B_{n}\left(s, t, q, Y_{0}, Y_{1}, 1\right)$ is given by

$$
\begin{aligned}
\sum_{n \geq 0}(1 & +t) B_{n}\left(s, t, q, Y_{0}, Y_{1}, 1\right) \frac{u^{n}}{\left(t^{2} ; q^{2}\right)_{n+1}} \\
& =\sum_{r \geq 0} t^{r} \frac{\left(u ; q^{2}\right)_{\lfloor r / 2\rfloor+1}}{\left(u Y_{0} ; q^{2}\right)_{\lfloor r / 2\rfloor+1}} \frac{\left(-u s q Y_{1} ; q^{2}\right)_{\lfloor(r+1) / 2\rfloor}}{\left(-u s q ; q^{2}\right)_{\lfloor(r+1) / 2\rfloor}} F_{r}(u ; s, q, 1),
\end{aligned}
$$

where $F_{r}(u ; s, q, 1)$ is given by

$$
\begin{aligned}
& F_{r}(u ; s, q, 1)=\frac{\left(u s^{2} q^{2} ; q^{2}\right)_{\lfloor r / 2\rfloor}}{\left(u ; q^{2}\right)_{\lfloor r / 2\rfloor+1}} \\
& \times \frac{(1-s q)\left(u ; q^{2}\right)_{\lfloor(r+1) / 2\rfloor}\left(u s q ; q^{2}\right)_{\lfloor r / 2\rfloor}}{\left(u ; q^{2}\right)_{\lfloor(r+1) / 2\rfloor}\left(u s q ; q^{2}\right)_{\lfloor r / 2\rfloor}-s q\left(u s q ; q^{2}\right)_{\lfloor(r+1) / 2\rfloor}\left(u s^{2} q^{2} ; q^{2}\right)\lfloor r / 2\rfloor} .
\end{aligned}
$$

Corollary 1.4. The factorial generating function for the polynomials $B_{n}\left(s, 1, q, Y_{0}, Y_{1}, 1\right)$ is given by

$$
\begin{aligned}
\sum_{n \geq 0} B_{n}\left(s, 1, q, Y_{0}, Y_{1}, 1\right) \frac{u^{n}}{\left(q^{2} ; q^{2}\right)_{n}} & \\
= & \frac{e_{q^{2}}\left(u Y_{0}\right) E_{q^{2}}\left(u s q Y_{1}\right)}{E_{q^{2}}(u s q)} \frac{(1-s q)}{e_{q^{2}}\left(u s^{2} q^{2}\right)-s q e_{q^{2}}(u)} .
\end{aligned}
$$




\section{A SEXTUPLE DISTRIBUTION}

The proof of Theorem 1.1 requires several steps. First, the factorial generating function for the polynomials $B_{n}\left(s, t, q, Y_{0}, Y_{1}, Z\right)$, as it appears on the left-hand side of (1.4), is shown to be equal to a series $\sum_{r \geq 0} t^{r} a_{r}$,
where each $a_{r}$ is the product of the rational function

$$
\frac{\left(u ; q^{2}\right)_{\lfloor r / 2\rfloor+1}}{\left(u Y_{0} ; q^{2}\right)_{\lfloor r / 2\rfloor+1}} \frac{\left(-u s q Y_{1} Z ; q^{2}\right)_{\lfloor(r+1) / 2\rfloor}}{\left(-u s q Z ; q^{2}\right)_{\lfloor(r+1) / 2\rfloor}}
$$

by the generating series $\sum u^{\lambda w} s^{\text {fexc } w} q^{\operatorname{tot} c} Z^{\text {neg } w}$ for the set $\operatorname{WSP}(r)$ of the so-called weighted signed permutations by a certain four-variable statistic $(\lambda$, tot, fexc, neg) (see Theorem 4.2). The combinatorics of the weighted signed permutations was introduced in our previous paper [FoHa07a], but this time the extra variable " $s$ " is to be added to make the calculation. All this derivation is developed in Sections 2 and 3. Noticeably, the variables $Y_{0}$ and $Y_{1}$ that carry the information on fixed points occur only in the above fraction, but not in the latter generating series. Also, the positive fixed point counter $Y_{0}$ occurs in the denominator of that fraction, while the negative fixed point counter $Y_{1}$ only in the numerator.

The next step is to show that this generating series for weighted signed permutations is also equal to the generating series for words, whose letters belong to the interval $[0, r]$, by another four-variable statistic $(\lambda$, tot $v$, evdec + odd, odd). This is achieved by means of the construction of a bijection of the set of weighted signed permutations onto the set of those words having the adequate properties (see Theorem 5.1).

The crucial calculation is to evaluate the latter generating function and show that it is equal to the expression $F_{r}(u ; s, q, Z)$ displayed in (1.5). Two proofs are given, the first one using a $V$-word decomposition theorem (see Theorem 6.1) derived in [FoHa07b] together with the traditional qseries telescoping technique, the second one taking advantage of a word factorization, which consists of cutting each word after every odd letter (see Section 8).

Formula (1.6) (resp. (1.9)) is deduced from (1.4) (resp. from (1.7)) by the traditional token that consists of multiplying the latter one by $(1-t)$ and letting $r$ tend to $+\infty$, so that Corollaries 1.2 and 1.4 are easy consequences of Theorem 1.1 and Corollary 1.3.

We prove Corollary 1.3 in two different ways: first, as a specialization of Theorem 1.1 by an evaluation of the fraction $F_{r}(u ; s, q, Z)$ for $Z=1$ (this requires some manipulations on $q$-series), second, by showing directly that $F_{r}(u ; s, q, 1)$ is the generating function for words by the two-variable statistic (tot, 2 evdec + odd), using a bijection constructed in our previous paper $[\mathrm{FoHa07b}$. We end the paper by deriving several specializations of Theorem 1.1, in particular, the joint distribution of the pair (fexc, fdes) over the group $B_{n}$. 


\section{DOMINIQUE FOATA AND GUO-NIU HAN}

\section{Weighted signed permutations}

This section will appear to be an updated version of Section 4 of our previous paper [FoHa07a], where the notion of weighted signed permutation was introduced. With the addition of "fexc" it was essential to ascertain how that statistic behaved in the underlying combinatorial construction.

We use the following notations: if $c=c_{1} c_{2} \cdots c_{n}$ is a word, whose letters are nonnegative integers, let $\lambda(c):=n$ be the length of $c$, tot $c:=$ $c_{1}+c_{2}+\cdots+c_{n}$ the sum of its letters and odd $c$ the number of its odd letters. Furthermore, $\mathrm{NIW}_{n}\left(\right.$ resp. $\mathrm{NIW}_{n}(r)$ ) designates the set of all nonincreasing monotonic words of length $n$, whose letters are nonnegative integers (resp. nonnegative integers at most equal to $r)$. Also let $\mathrm{NIW}_{n}^{e}(r)\left(\operatorname{resp} . \mathrm{DW}_{n}^{o}(r)\right)$ be the subset of $\operatorname{NIW}_{n}(r)$ of the monotonic nonincreasing (resp. strictly decreasing) words all letters of which are even (resp. odd).

Next, each pair $\left(\begin{array}{c}c \\ w\end{array}\right)$ is called a weighted signed permutation of order $n$ if the four properties (wsp1)-(wsp4) hold:

(wsp1) $c$ is a word $c_{1} c_{2} \cdots c_{n}$ from $\mathrm{NIW}_{n}$;

(wsp2) $w$ is a signed permutation $x_{1} x_{2} \cdots x_{n}$ from $B_{n}$;

(wsp3) $c_{k}=c_{k+1} \Rightarrow x_{k}<x_{k+1}$ for all $k=1,2, \ldots, n-1$;

(wsp4) $x_{k}$ is positive (resp. negative) whenever $c_{k}$ is even (resp. odd).

When $w$ has no fixed points, either negative or positive, we say that $\left(\begin{array}{l}c \\ w\end{array}\right)$ is a weighted signed derangement. The set of weighted signed permutations (resp. derangements) $\left(\begin{array}{c}c \\ w\end{array}\right)=\left(\begin{array}{c}c_{1} c_{2} \cdots c_{n} \\ x_{1} x_{2} \cdots x_{n}\end{array}\right)$ of order $n$ is denoted by $\mathrm{WSP}_{n}$ (resp. by $\mathrm{WSD}_{n}$ ). The subset of all those weighted signed permutations (resp. derangements) such that $c_{1} \leq r$ is denoted by $\operatorname{WSP}_{n}(r)\left(\right.$ resp. by $\left.\operatorname{WSD}_{n}(r)\right)$.

For example, the following pair

$$
\left(\begin{array}{c}
c \\
w
\end{array}\right)=\left(\begin{array}{cc|c|ccc|ccc|r|rr|r}
1 & 2 & 3 & 4 & 5 & 6 & 7 & 8 & 9 & 10 & 11 & 12 & 13 \\
10 & 10 & 9 & \overline{7} & 7 & 7 & 4 & 4 & 4 & 3 & 2 & 2 & 1 \\
\mathbf{1} & \mathbf{2} & \overline{7} & \overline{6} & \overline{5} & \overline{4} & 3 & \mathbf{8} & \mathbf{9} & \overline{\mathbf{1 0}} & \underline{12} & \underline{13} & \frac{11}{11}
\end{array}\right)
$$

is a weighted signed permutation of order 13. It has four positive fixed points $(\mathbf{1}, \mathbf{2}, \mathbf{8}, \mathbf{9})$, two negative fixed points $(\overline{\mathbf{5}}, \overline{\mathbf{1 0}})$ and two excedances $(\underline{12}, \underline{13})$.

Proposition 2.1. With each weighted signed permutation $\left(\begin{array}{c}c \\ w\end{array}\right)$ from the set $\operatorname{WSP}_{n}(r)$ can be associated a unique sequence $\left(i, j, k,\left(\begin{array}{c}c^{\prime} \\ w^{\prime}\end{array}\right), v^{e}, v^{o}\right)$ such that

(1) $i, j, k$ are nonnegative integers of sum $n$;

(2) $\left(\begin{array}{c}c^{\prime} \\ w^{\prime}\end{array}\right)$ is a weighted signed derangement from the set $\operatorname{WSD}_{i}(r)$;

(3) $v^{e}$ is a nonincreasing word with even letters from the set $\operatorname{NIW}_{j}^{e}(r)$;

(4) $v^{o}$ is a decreasing word with odd letters from the set $\mathrm{DW}_{k}^{o}(r)$; 


\section{A SEXTUPLE DISTRIBUTION}

having the following properties:

$$
\begin{aligned}
\operatorname{tot} c=\operatorname{tot} c^{\prime}+\operatorname{tot} v^{e}+\operatorname{tot} v^{o} ; & \operatorname{neg} w=\operatorname{neg} w^{\prime}+\lambda\left(v^{o}\right) ; \\
\operatorname{fix}^{+} w=\lambda\left(v^{e}\right) ; \quad \operatorname{fix}^{-} w=\lambda\left(v^{o}\right) ; & \operatorname{fexc} w=\operatorname{fexc} w^{\prime}+\lambda\left(v^{0}\right) .
\end{aligned}
$$

The bijection $\left(\begin{array}{c}c \\ w\end{array}\right) \mapsto\left(\left(\begin{array}{c}c^{\prime} \\ w^{\prime}\end{array}\right), v^{e}, v^{o}\right)$ is quite natural to define. Only its reverse requires some attention. To get the latter three-term sequence from $\left(\begin{array}{l}c \\ w\end{array}\right)$ proceed as follows:

(a) let $l_{1}, \ldots, l_{\alpha}$ (resp. $\left.m_{1}, \ldots, m_{\beta}\right)$ be the increasing sequence of the integers $l_{i}$ (resp. $m_{i}$ ) such that $x_{l_{i}}$ (resp. $x_{m_{i}}$ ) is a positive (resp. negative) fixed point of $w$;

(b) define: $v^{e}:=c_{l_{1}} \cdots c_{l_{\alpha}}$ and $v^{o}:=c_{m_{1}} \cdots c_{m_{\beta}}$;

(c) remove all the columns $\left(\begin{array}{c}c_{l_{1}} \\ x_{l_{1}}\end{array}\right), \ldots,\left(\begin{array}{c}c_{l_{\alpha}} \\ x_{l_{\alpha}}\end{array}\right),\left(\begin{array}{c}c_{m_{1}} \\ x_{m_{1}}\end{array}\right), \ldots,\left(\begin{array}{c}c_{m_{\beta}} \\ x_{m_{\beta}}\end{array}\right)$ from $\left(\begin{array}{c}c \\ w\end{array}\right)$ and let $c^{\prime}$ be the nonincreasing word derived from $c$ after the removal;

(d) once the letters $x_{l_{1}}, \ldots, x_{l_{\alpha}}, x_{m_{1}}, \ldots, x_{m_{\beta}}$ have been removed from the signed permutation $w$ the remaining ones form a signed permutation of a subset $A$ of $[n]$, of cardinality $n-\alpha-\beta$. Using the unique increasing bijection $\phi$ of $A$ onto the interval $[n-\alpha-\beta]$ replace each remaining letter $x_{i}$ by $\phi\left(x_{i}\right)$ if $x_{i}>0$ or by $-\phi\left(-x_{i}\right)$ if $x_{i}<0$. Let $w^{\prime}$ be the signed derangement of order $n-\alpha-\beta$ thereby obtained. There is no difficulty verifying that the properties listed in (2.1) hold.

For instance, with the above weighted signed permutation we have: $v^{e}=10,10,4,4$ and $v^{o}=7,3$. After removing the fixed point columns we obtain:

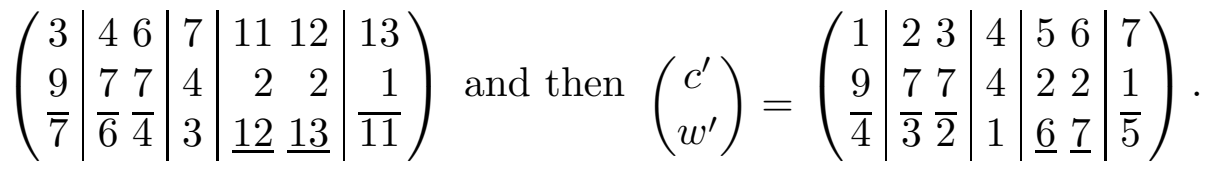

The signed permutation $w\left(\right.$ resp. $\left.w^{\prime}\right)$ has two excedances $\underline{12}, \underline{13}(\operatorname{resp} . \underline{6}, \underline{7})$ and six (resp. four) negative letters. Furthermore, $v^{0}=7,3$ is of length 2 . Hence fexc $w=2 \operatorname{exc} w+\operatorname{neg} w=2 \times 2+6=10=2 \times 2+4+2=$ fexc $w^{\prime}+\lambda\left(v^{0}\right)$.

For reconstructing $\left(\begin{array}{c}c \\ w\end{array}\right)$ from the sequence $\left(\left(\begin{array}{c}c^{\prime} \\ w^{\prime}\end{array}\right), v^{e}, v^{o}\right)$ consider the nonincreasing rearrangement of the juxtaposition product $v^{e} v^{o}$ in the form $b_{1}^{h_{1}} \cdots b_{m}^{h_{m}}$, where $b_{1}>\cdots>b_{m}$ and $h_{i} \geq 1$ (resp. $\left.h_{i}=1\right)$ if $b_{i}$ is even (resp. odd). The pair $\left(\begin{array}{c}c^{\prime} \\ w^{\prime}\end{array}\right)$ being decomposed into matrix blocks, as shown in the example, each letter $b_{i}$ indicates where the $h_{i}$ fixed point columns are to be inserted. We do not give more details and simply illustrate the construction with the running example.

With the previous example $b_{1}^{h_{1}} \cdots b_{m}^{h_{m}}=10^{2} 74^{2} 3$. First, implement $10^{2}$ :

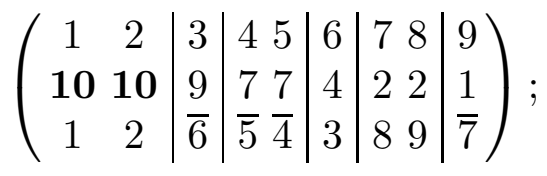


then 7 :

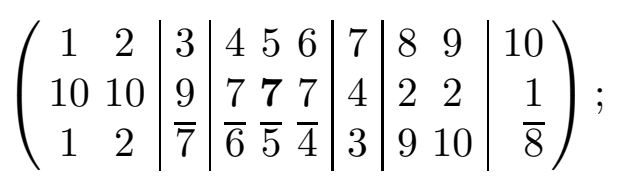

notice that because of condition (wsp3) the letter $\mathbf{7}$ is to be inserted in second position in the third block; then insert $4^{2}$ :

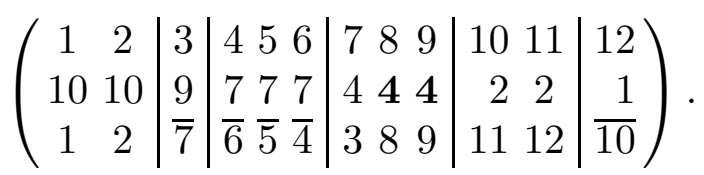

The implementation of 3 gives back the original weighted signed permutation $\left(\begin{array}{c}c \\ w\end{array}\right)$.

\section{A summation on weighted signed permutations}

For $0 \leq k \leq n$ let $\left[\begin{array}{l}n \\ k\end{array}\right]_{q}:=\frac{(q ; q)_{n}}{(q ; q)_{k}(q ; q)_{n-k}}$ be the usual $q$-binomial coefficient. It is $q$-routine (see, e.g., [An76, chap. 3]) to prove the following identities, where $v_{1}$ is the first letter of $v$ :

$$
\begin{gathered}
\frac{1}{(u ; q)_{N}}=\sum_{n \geq 0}\left[\begin{array}{c}
N+n-1 \\
n
\end{array}\right]_{q} u^{n} ; \quad\left[\begin{array}{c}
N+n \\
n
\end{array}\right]_{q}=\sum_{v \in \mathrm{NIW}_{n}(N)} q^{\operatorname{tot} v} ; \\
\frac{1}{(u ; q)_{N+1}}=\sum_{n \geq 0} u^{n} \sum_{v \in \mathrm{NIW}_{n}(N)} q^{\operatorname{tot} v}=\frac{1}{1-u} \sum_{v \in \mathrm{NIW}_{n}} q^{\operatorname{tot} v} u^{v_{1}} ; \\
\frac{1}{\left(u ; q^{2}\right)_{\lfloor r / 2\rfloor+1}}=\sum_{n \geq 0} u^{n} \sum_{v^{e} \in \mathrm{NIW}_{n}^{e}(r)} q^{\operatorname{tot} v^{e}} ; \\
\left(-u q ; q^{2}\right)_{\lfloor(r+1) / 2\rfloor}=\sum_{n \geq 0} u^{n} \sum_{v^{o} \in \operatorname{DW}_{n}^{o}(r)} q^{\operatorname{tot} v^{o}} .
\end{gathered}
$$

The last two formulas and Proposition 2.1 are now used to calculate the generating function for the weighted signed permutations. The symbols $\mathrm{NIW}^{e}(r), \mathrm{DW}^{o}(r), \operatorname{WSP}(r), \operatorname{WSD}(r)$ designate the unions for $n \geq 0$ of the corresponding symbols with an $n$-subscript.

Proposition 3.1. The following identity holds:

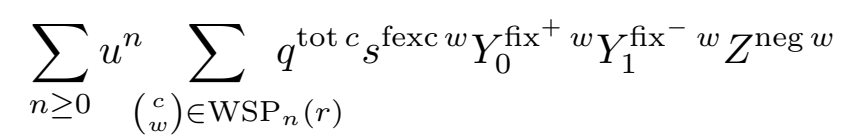

$$
=\frac{\left(u ; q^{2}\right)_{\lfloor r / 2\rfloor+1}}{\left(u Y_{0} ; q^{2}\right)_{\lfloor r / 2\rfloor+1}} \frac{\left(-u s q Y_{1} Z ; q^{2}\right)_{\lfloor(r+1) / 2\rfloor}}{\left(-u s q Z ; q^{2}\right)_{\lfloor(r+1) / 2\rfloor}} \times \sum_{n \geq 0} u^{n} \sum_{\left(\begin{array}{c}
c \\
w
\end{array}\right) \in \mathrm{WSP}_{n}(r)} q^{\operatorname{tot} c} s^{\mathrm{fexc} w} Z^{\mathrm{neg} w} .
$$




\section{A SEXTUPLE DISTRIBUTION}

Proof. First, summing over $\left(w^{e}, w^{o},\left(\begin{array}{c}c \\ w\end{array}\right)\right) \in \operatorname{NIW}^{e}(r) \times \mathrm{DW}^{o}(r) \times \mathrm{WSP}(r)$, we have

$$
\begin{aligned}
& \sum_{w^{e}, w^{o},\left(\begin{array}{c}
c \\
w
\end{array}\right)} u^{\lambda\left(w^{e}\right)} q^{\operatorname{tot} w^{e}} \times(u s Z)^{\lambda\left(w^{o}\right)} q^{\operatorname{tot} w^{o}} \times u^{\lambda(c)} q^{\operatorname{tot} c} s^{\text {fexc } w} Y_{0}^{\mathrm{fix}^{+} w} Y_{1}^{\mathrm{fix}^{-} w} Z^{\text {neg } w} \\
& (3.4) \quad=\frac{\left(-u s q Z ; q^{2}\right)\lfloor(r+1) / 2\rfloor}{\left(u ; q^{2}\right)\lfloor r / 2\rfloor+1} \times \sum_{\left(\begin{array}{c}
c \\
w
\end{array}\right)} u^{\lambda(c)} q^{\operatorname{tot} c} s^{\text {fexc } w} Y_{0}^{\mathrm{fix}^{+} w} Y_{1}^{\mathrm{fix}{ }^{-} w} Z^{\text {neg } w}
\end{aligned}
$$

by (3.1) and (3.2). As fexc $w=$ fexc $w^{\prime}+\lambda\left(v^{o}\right)$, Proposition 2.1 implies that the initial expression can also be summed over five-term sequences $\left(\left(\begin{array}{c}c^{\prime} \\ w^{\prime}\end{array}\right), v^{e}, v^{o}, w^{e}, w^{o}\right)$ from $\operatorname{WSD}(r) \times \mathrm{NIW}^{e}(r) \times \mathrm{DW}^{o}(r) \times \mathrm{NIW}^{e}(r) \times \mathrm{DW}^{o}(r)$ in the form

$$
\begin{gathered}
\sum_{\left.\begin{array}{c}
c^{\prime} \\
w^{\prime}
\end{array}\right), v^{e}, v^{o}, w^{e}, w^{o}} u^{\lambda\left(c^{\prime}\right)} q^{\operatorname{tot} c^{\prime}} s^{\text {fexc } w^{\prime}} Z^{\operatorname{neg} w^{\prime}} \times\left(u Y_{0}\right)^{\lambda\left(v^{e}\right)} q^{\operatorname{tot} v^{e}} \times\left(u s Y_{1} Z\right)^{\lambda\left(v^{o}\right)} q^{\operatorname{tot} v^{o}} \\
\times u^{\lambda\left(w^{e}\right)} q^{\operatorname{tot} w^{e}} \times(u s Z)^{\lambda\left(w^{o}\right)} q^{\operatorname{tot} w^{o}} \\
=\sum_{v^{e}, v^{o}}\left(u Y_{0}\right)^{\lambda\left(v^{e}\right)} q^{\operatorname{tot} v^{e}} \times\left(u s Y_{1} Z\right)^{\lambda\left(v^{o}\right)} q^{\operatorname{tot} v^{o}} \\
\times \sum_{\left(\begin{array}{c}
c^{\prime} \\
w^{\prime}
\end{array}\right), w^{e}, w^{o}} u^{\lambda\left(c^{\prime}\right)} q^{\operatorname{tot} c^{\prime}} s^{\text {fexc } w^{\prime}} Z^{\text {neg } w^{\prime}} \times u^{\lambda\left(w^{e}\right)} q^{\operatorname{tot} w^{e}} \times(u s Z)^{\lambda\left(w^{o}\right)} q^{\operatorname{tot} w^{o}} .
\end{gathered}
$$

The first summation can be evaluated by (3.1) and (3.2), while by Proposition 2.1 again the second sum can be expressed as a sum over weighted signed permutations $\left(\begin{array}{c}c \\ w\end{array}\right) \in \operatorname{WSP}(r)$. Therefore, the initial sum is also equal to

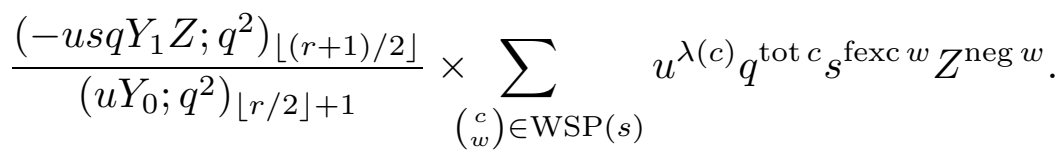

Identity (3.3) follows by equating (3.4) with (3.5).

\section{A further evaluation}

Proposition 4.1. Let $B_{n}\left(s, t, q, Y_{0}, Y_{1}, Z\right)$ denote the generating polynomial for the group $B_{n}$ by the statistic (fexc, fdes, maj, fix ${ }^{+}, f_{i x}{ }^{-}$, neg). Then

$$
\begin{aligned}
& \frac{1+t}{\left(t^{2} ; q^{2}\right)_{n+1}} B_{n}\left(s, t, q, Y_{0}, Y_{1}, Z\right) \\
& =\sum_{r \geq 0} t^{r} \sum_{\left(\begin{array}{l}
c \\
w
\end{array}\right) \in \operatorname{WSP}_{n}(r)} q^{\text {tot } c} s^{\text {fexc } w} Y_{0}^{\mathrm{fix}^{+} w} Y_{1}^{\mathrm{fix}-w} Z^{\text {neg } w} .
\end{aligned}
$$




\section{DOMINIQUE FOATA AND GUO-NIU HAN}

Proof. A very similar calculation has been made in the proof of Theorem 4.1 in [FoHa05]. We also make use of the identities on the $q$ ascending factorials that were recalled in the previous section. First,

$$
\begin{aligned}
\frac{1+t}{\left(t^{2} ; q^{2}\right)_{n+1}} & =\sum_{r^{\prime} \geq 0}\left(t^{2 r^{\prime}}+t^{2 r^{\prime}+1}\right)\left[\begin{array}{c}
n+r^{\prime} \\
r^{\prime}
\end{array}\right]_{q^{2}} \\
& =\sum_{r \geq 0} t^{r}\left[\begin{array}{c}
n+\lfloor r / 2\rfloor \\
\lfloor r / 2\rfloor
\end{array}\right]_{q^{2}}=\sum_{r \geq 0} t^{r} \sum_{b \in \mathrm{NIW}_{n}(\lfloor r / 2\rfloor)} q^{2 \operatorname{tot} b} .
\end{aligned}
$$

Then,

$$
\begin{aligned}
& \frac{1+t}{\left(t^{2} ; q^{2}\right)_{n+1}} B_{n}\left(s, t, q, Y_{0}, Y_{1}, Z\right)
\end{aligned}
$$

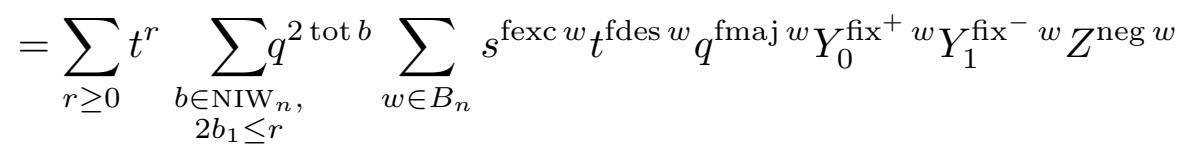

$$
\begin{aligned}
& =\sum_{r \geq 0} t^{r} \sum_{\substack{b \in \mathrm{NIW}_{n}, w \in B_{n} \\
2 b_{1}+\text { fdes } w \leq r}} s^{\text {fexc } w} q^{2 \operatorname{tot} b+\mathrm{fmaj} w} Y_{0}^{\mathrm{fix}{ }^{+} w} Y_{1}^{\mathrm{fix}-w} Z^{\text {neg } w} .
\end{aligned}
$$

As proved in $[$ FoHa05, $\S 4]$ to each $\left(\begin{array}{c}c \\ w\end{array}\right)=\left(\begin{array}{c}c_{1} \cdots c_{n} \\ x_{1} \cdots x_{n}\end{array}\right) \in \operatorname{WSP}_{n}(s)$ there corresponds a unique $b=b_{1} \cdots b_{n} \in \mathrm{NIW}_{n}$ such that $2 b_{1}+$ fdes $w=c_{1}$ and 2 tot $b+$ fmaj $w=$ tot $c$. Moreover, the mapping $\left(\begin{array}{c}c \\ w\end{array}\right) \mapsto(b, w)$ is a bijection of $\operatorname{WSP}_{n}(r)$ onto the set of all pairs $(b, w)$ such that $b=b_{1} \cdots b_{n} \in \mathrm{NIW}_{n}$ and $w \in B_{n}$ with the property that $2 b_{1}+$ fdes $w \leq r$.

The word $b$ is determined as follows: write the signed permutation $w$ as a linear word $w=x_{1} x_{2} \ldots x_{n}$ and for each $k=1,2, \ldots, n$ let $z_{k}$ be the number of descents $\left(x_{i}>x_{i+1}\right)$ in the right factor $x_{k} x_{k+1} \cdots x_{n}$ and let $\epsilon_{k}$ be equal to 0 or 1 depending on whether $x_{k}$ is positive or negative. Also for each $k=1,2, \ldots, n$ define $a_{k}:=\left(c_{k}-\epsilon_{k}\right) / 2, b_{k}:=\left(a_{k}-z_{k}\right)$ and form the word $b=b_{1} \cdots b_{n}$.

For example,

$$
\begin{aligned}
& \text { Id }=12345567889910 \\
& c=9774442211 \\
& w=\overline{4} \overline{3} \overline{2} 15689 \overline{10} \overline{7} \\
& z=1111111100 \\
& \epsilon=1111100000001 \\
& a=4332221100 \\
& b=3221110000
\end{aligned}
$$

Pursuing the above calculation we get (4.1).

The next theorem is then a consequence of Propositions 3.1 and 4.1. 


\section{A SEXTUPLE DISTRIBUTION}

Theorem 4.2. The following identity holds:

$$
\begin{aligned}
& \sum_{n \geq 0}(1+t) B_{n}\left(s, t, q, Y_{0}, Y_{1}, Z\right) \frac{u^{n}}{\left(t^{2} ; q^{2}\right)_{n+1}} \\
& =\sum_{r \geq 0} t^{r} \frac{\left(u ; q^{2}\right)_{\lfloor r / 2\rfloor+1}}{\left(u Y_{0} ; q^{2}\right)_{\lfloor r / 2\rfloor+1}} \frac{\left(-u s q Y_{1} Z ; q^{2}\right)_{\lfloor(r+1) / 2\rfloor}}{\left(-u s q Z ; q^{2}\right)_{\lfloor(r+1) / 2\rfloor}} \sum_{\left.\begin{array}{c}
c \\
w
\end{array}\right) \in \operatorname{WSP}(r)} u^{\lambda w} q^{\operatorname{tot} c} s^{\text {fexc } w} Z^{\text {neg } w} .
\end{aligned}
$$

In view of the statements of Theorems 1.1 and 4.2 we see that the former theorem will be proved if we can show that the following identity holds:

$$
\sum_{\left(\begin{array}{c}
c \\
w
\end{array}\right) \in \operatorname{WSP}(r)} u^{\lambda w} q^{\operatorname{tot} c} s^{\text {fexc } w} Z^{\text {neg } w}=F_{r}(u ; s, q, Z),
$$

with $F_{r}(u ; s, q, Z)$ given by (1.5). In our paper [FoHa06a] the sum $\sum q^{\text {tot } c} Z^{\text {neg } w} \quad\left(\left(\begin{array}{c}c \\ w\end{array}\right) \in \mathrm{WSP}_{n}(r)\right)$ has been calculated, by setting up a bijection of $\operatorname{WSP}_{n}(r)$ onto the set $[0, r]^{n}$ of the words of length $n$, whose letters are taken from the interval $[0, r]$. Because of the presence of the new statistic "fexc" another bijection is to be constructed. If $v$ is the image of $\left(\begin{array}{c}c \\ w\end{array}\right)$ under the new bijection, then the statistic "fexc" on signed permutations must have a counterpart on words. This role is played by the so-called number of even decreases, as shown in the next section.

\section{Even decreases on words}

Say that a letter $y_{i}$ of a word $v=y_{1} y_{2} \cdots y_{n}$ from $[0, r]^{n}$ is a decrease in $v$ if $1 \leq i \leq n-1$ and $y_{i} \geq y_{i+1} \geq \cdots \geq y_{j}>y_{j+1}$ for some $j$ such that $i \leq j \leq n-1$. Let $\operatorname{dec} v$ (resp. evdec $v$ ) denote the number of decreases (resp. of even decreases) in $v$. Notice that $\operatorname{dec} v \geq \operatorname{des} v$ (the number of descents in $v$ ) and $\operatorname{dec} v=\operatorname{des} v$ whenever $v$ is a permutation (without repetitions). Also, let odd $v$ be the number of odd letters in $v$.

Recall that a nonempty word $v=y_{1} y_{2} \cdots y_{n}$ is a Lyndon word, if either $n=1$, or $n \geq 2$ and, with respect to the lexicographic order, the inequality $y_{1} y_{2} \cdots y_{n}>y_{i} y_{i+1} \cdots y_{n} y_{1} \cdots y_{i-1}$ holds for every $i$ such that $2 \leq i \leq n$. Let $v, v^{\prime}$ be two nonempty primitive words (none of them can be written as $v_{0}^{a}$ for $a \geq 2$ and some word $v_{0}$ ). We write $v \preceq v^{\prime}$ if and only if $v^{a} \leq v^{\prime a}$ with respect to the lexicographic order for an integer $a$ large enough. As shown for instance in [Lo83, Theorem 5.1.5] (also see [Ch58], [Sch65]) each nonempty word $v$ can be written uniquely as a product $l_{1} l_{2} \cdots l_{k}$, called its Lyndon factorization, where each $l_{i}$ is a Lyndon word and $l_{1} \preceq l_{2} \preceq \cdots \preceq l_{k}$. In the example below the Lyndon factorization of $v$ has been materialized by vertical bars. The essential property of the Lyndon factorization needed in the sequel is the following:

$$
\operatorname{dec} v=\operatorname{dec} l_{1}+\operatorname{dec} l_{2}+\cdots+\operatorname{dec} l_{k} .
$$




\section{DOMINIQUE FOATA AND GUO-NIU HAN}

Now, start with the Lyndon factorization $l_{1} l_{2} \cdots l_{k}$ of a word $v$ from $[0, r]^{n}$. With such a $v$ we associate a permutation $\sigma$ from $\mathfrak{S}_{n}$ by means of a procedure developed by Gessel-Reutenauer [GeRe93]: each letter $y_{i}$ of $v$ belongs to a Lyndon word factor $l_{h}$, so that $l_{h}=v^{\prime} y_{i} v^{\prime \prime}$. Then, form the infinite word $A\left(y_{i}\right):=y_{i} v^{\prime \prime} v^{\prime} y_{i} v^{\prime \prime} v^{\prime} \ldots$ If $y_{i}$ and $y_{i^{\prime}}$ are two letters of $v$, say that $y_{i}$ precedes $y_{i^{\prime}}$ if $A\left(y_{i}\right)>A\left(y_{i^{\prime}}\right)$ for the lexicographic order, or if $A\left(y_{i}\right)=A\left(y_{i^{\prime}}\right)$ and $y_{i}$ is to the right of $y_{i^{\prime}}$ in the word $v$. This precedence determines a total order on the $n$ letters of $v$. The letter that precedes all the other ones is given label 1 , the next one label 2 , and so on. When each letter $y_{i}$ of $v$ is replaced by its label, say, lab $\left(y_{i}\right)$, each Lyndon word factor $l_{j}$ becomes a new word $\tau_{j}$. The essential property is that each $\tau_{j}$ starts with its minimum element and those minimum elements read from left to right are in decreasing order. We can then interpret each $\tau_{j}$ as the cycle of a permutation and the (juxtaposition) product $\tau_{1} \tau_{2} \cdots \tau_{k}$ as the (functional) product of disjoint cycles. This product, said to be written in canonical form, defines a unique permutation $\sigma$ from $\mathfrak{S}_{n}([\operatorname{Lo} 83], \S 10.2)$.

For example,

$$
\begin{array}{l|cccc|c|c|ccccccc|ccccccc|c}
v=2 & 3 & 2 & 1 & 1 & 3 & 5 & 6 & 4 & 2 & 1 & 3 & 2 & 3 & 6 & 6 & 3 & 1 & 6 & 6 & 2 & 6 \\
\sigma=16 & 12 & 18 & 22 & 21 & 10 & 7 & 4 & 8 & 17 & 20 & 11 & 15 & 9 & 2 & 5 & 13 & 19 & 3 & 6 & 14 & 1
\end{array}
$$

The labels on the second row are obtained as follows: read the letters equal to 6 (the maximal letter) from left to right and form their associated infinite words: $64213236421 \cdots, 66316626631 \cdots, 6316621131 \cdots$, $662663166 \cdots, 62663166 \cdots, 66666 \cdots$ Those letters 6 read from left to right will be given the labels $4,2,5,3,6,1$. We continue the labellings by reading the letters equal to 5 , then $4, \ldots$ in the above word $v$.

No decrease $y_{i}$ in $v$ can be the rightmost letter of a Lyndon word factor $l_{h}$. We have then $l_{h}=\cdots y_{i} y_{i+1} \cdots y_{j} y_{j+1} \cdots$ with $y_{i} \geq y_{i+1} \geq$ $\cdots \geq y_{j}>y_{j+1}$. Consequently, $A\left(y_{i}\right)>A\left(y_{i+1}\right)$ and $\operatorname{lab}\left(y_{i}\right)<\operatorname{lab}\left(y_{i+1}\right)$. Conversely, if lab $\left(y_{i}\right)<\operatorname{lab}\left(y_{i+1}\right)$ and $y_{i}, y_{i+1}$ belong to the same Lyndon factor, then $y_{i}$ is a decrease in $v$. To each decrease $y_{i}$ in $v$ there corresponds a unique cycle $\tau_{h}$ of $\sigma$ and a pair lab $\left(y_{i}\right) \operatorname{lab}\left(y_{i+1}\right)$ of successive letters of $\tau_{h}$ such that $\operatorname{lab}\left(y_{i}\right)<\operatorname{lab}\left(y_{i+1}\right)$ and $\operatorname{lab}\left(y_{i+1}\right)=\sigma\left(\operatorname{lab}\left(y_{i}\right)\right)$.

Consider the monotonic nonincreasing rearrangement $c=c_{1} c_{2} \cdots c_{n}$ of $v$ and form the three-row matrix

$$
\begin{array}{cccc}
1 & 2 & \cdots & n \\
c_{1} & c_{2} & \cdots & c_{n} \\
\sigma(1) & \sigma(2) & \cdots & \sigma(n)
\end{array}
$$

Then, if $y_{i}$ is a decrease in $v$, the lab $\left(y_{i}\right)$-th column of the previous matrix is of the form $\quad \operatorname{lab}\left(y_{i}\right)$

$$
\underset{\operatorname{lab}\left(y_{i+1}\right)}{y_{i}} \text { with } \quad \operatorname{lab}\left(y_{i}\right)<\operatorname{lab}\left(y_{i+1}\right) .
$$




\section{A SEXTUPLE DISTRIBUTION}

At this stage we have $\operatorname{dec} v=\operatorname{exc} \sigma$. We then have to transform $\sigma$ into a signed permutation $w$ in such a way that only the excedances corresponding to the even decreases of $v$ are preserved. We proceed as follows. The word $c$ can be expressed as $a_{1}^{m_{1}} a_{2}^{m_{2}} \cdots a_{k}^{m_{k}}$ where $a_{1}>a_{2}>$ $\cdots>a_{k} \geq 0$ and $m_{1} \geq 1, m_{2} \geq 1, \ldots, m_{k} \geq 1$. We then define

$$
\begin{aligned}
x_{1} \cdots x_{m_{1}}:=\left\{\begin{array}{l}
\sigma(1) \cdots \sigma\left(m_{1}\right), \quad \text { if } a_{1} \text { is even; } \\
\bar{\sigma}\left(m_{1}\right) \cdots \bar{\sigma}(1), \quad \text { if } a_{1} \text { is odd; }
\end{array}\right. \\
x_{m_{1}+1} \cdots x_{m_{1}+m_{2}:}:=\left\{\begin{array}{l}
\sigma\left(m_{1}+1\right) \cdots \sigma\left(m_{1}+m_{2}\right), \quad \text { if } a_{2} \text { is even; } \\
\bar{\sigma}\left(m_{1}+m_{2}\right) \cdots \bar{\sigma}\left(m_{1}+1\right), \quad \text { if } a_{2} \text { is odd; }
\end{array}\right. \\
\cdots \\
\cdots \\
x_{m_{1}+\cdots+m_{k-1}+1} \cdots x_{n}:=\left\{\begin{array}{l}
\sigma\left(m_{1}+\cdots+m_{k-1}+1\right) \cdots \sigma(n), \text { if } a_{k} \text { is even; } \\
\bar{\sigma}(n) \cdots \bar{\sigma}\left(m_{1}+\cdots+m_{k-1}+1\right), \text { if } a_{k} \text { is odd }
\end{array}\right.
\end{aligned}
$$

The word $w:=x_{1} x_{2} \cdots x_{n}$ is then a signed permutation. When going from $\sigma$ to $w$, the excedances $\operatorname{lab}\left(y_{i}\right)<\operatorname{lab}\left(y_{i+1}\right)$ of $\sigma$ such that $y_{i}$ is odd have vanished. The other ones have been preserved. Hence, $2 \operatorname{evdec} v+\operatorname{odd} v=$ $2 \operatorname{exc} w+\operatorname{neg} w=$ fexc $w$. Finally, as $\sigma(i)>\sigma(i+1) \Rightarrow c_{i}>c_{i+1}$, the pair $\left(\begin{array}{c}c \\ w\end{array}\right)$ is a weighted signed permutation. We then have the desired bijection $[0, r]^{n} \rightarrow\left(\begin{array}{c}c \\ w\end{array}\right)$.

Theorem 5.1. The mapping $v \rightarrow\left(\begin{array}{c}c \\ w\end{array}\right)$ is a bijection of $[0, r]^{n}$ onto $\mathrm{WSP}_{n}(r)$ having the following properties:

$$
\operatorname{tot} c=\operatorname{tot} v ; \quad \operatorname{fexc} w=2 \operatorname{evdec} v+\operatorname{odd} v ; \quad \operatorname{neg} w=\operatorname{odd} v .
$$

With the running example we have

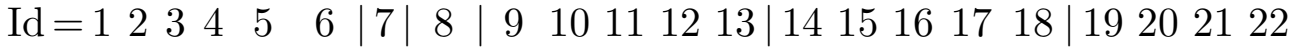

$$
\begin{aligned}
& c=6 \begin{array}{lllll|l|l|lllll|lllll|llll}
6 & 6 & 6 & 6 & 6 & 5 & 4 & 3 & 3 & 3 & 3 & 3 & 2 & 2 & 2 & 2 & 2 & 1 & 1 & 1 & 1
\end{array}
\end{aligned}
$$

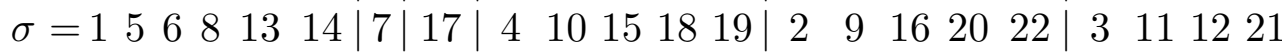

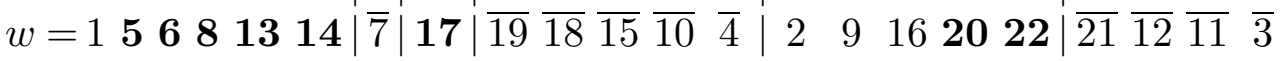

The signed permutation $w$ has eight excedances (reproduced in boldface) and ten negative letters. Therefore, fexc $w=2 \times 8+10=26$. There are eight even decreases of the word $v: 2 \rightarrow 1,6 \rightarrow 4,4 \rightarrow 2$, $2 \rightarrow 1,6 \rightarrow 6,6 \rightarrow 3,6 \rightarrow 6,6 \rightarrow 2$. Moreover, odd $v=10$. Hence $2 \operatorname{evdec} v+\operatorname{odd} v=26=$ fexc $w$ and of course neg $w=\operatorname{odd} v=10$.

In view of (4.3) and Theorem 5.1 the proof of Theorem 1.1 will be completed if the identy

$$
\sum_{v \in[0, r]^{*}} u^{\lambda v} q^{\operatorname{tot} v} s^{2 \text { evdec } v+\operatorname{odd} v} Z^{\text {odd } v}=F_{r}(u ; s, q, Z)
$$

holds, the sum being over all words whose letters are in $[0, r]$. 


\section{The calculation of the latter sum}

As introduced in our previous paper [FoHa07b] a word $w=x_{1} x_{2} \ldots x_{n}$ of length $n \geq 2$ is said to be a $V$-word, if for some integer $i$ such that $1 \leq i \leq n-1$ we have $x_{1} \geq x_{2} \geq \cdots \geq x_{i}>x_{i+1}$ and $x_{i+1} \leq x_{i+2} \leq$ $\cdots \leq x_{n}<x_{i}$ whenever $i+1<n$. The pair $\left(x_{i}, x_{i+1}\right)$ is called the critical biletter of $w$. The $V$-word decomposition for permutations was introduced by Kim and Zeng [KiZe01]. The following theorem is a simple consequence of Theorem 3.4 proved in our previous paper [FoHa07b].

Theorem 6.1 ( $V$-word decomposition). To each word $v=y_{1} y_{2} \cdots y_{n}$ whose letters are nonnegative integers there corresponds a unique sequence $\left(v_{0}, v_{1}, v_{2}, \ldots, v_{k}\right)$, where $v_{0}$ is a monotonic nondecreasing word and $v_{1}$, $v_{2}, \ldots, v_{k}$ are $V$-words with the further property that $v_{0} v_{1} v_{2} \cdots v_{k}$ is a rearrangement of $v$ and

$$
\operatorname{evdec} v=\operatorname{evdec} v_{1}+\operatorname{evdec} v_{2}+\cdots+\operatorname{evdec} v_{k} .
$$

Let $X_{0}, X_{1}, \ldots, X_{r}$ be $(r+1)$ commuting variables. The even weight, evweight $v$, of each word $v=y_{1} y_{2} \ldots y_{n}$ is defined to be

$$
\text { evweight } v:=X_{y_{1}} X_{y_{2}} \cdots X_{y_{n}} s^{2 \text { evdec } v} \text {. }
$$

Now, consider the infinite series:

$$
\begin{aligned}
U(l) & :=\prod_{l \leq j \leq r}\left(1-s^{2 \chi(j \text { even })} X_{j}\right)^{-1}, \quad(1 \leq l \leq r) ; \\
M(k, l) & :=\prod_{k \leq j \leq l-1}\left(1-X_{j}\right)^{-1}, \quad(0 \leq k<l) .
\end{aligned}
$$

Then, the generating function for $V$-words, whose critical biletter is $(l, k)$ $(0 \leq k<l \leq r)$, by "evweight" is equal to:

$$
U(l) s^{2 \chi(l \text { even })} X_{l} X_{k} M(k, l) \text {. }
$$

The following theorem is then a consequence of Theorem 6.1.

Theorem 6.2. We have the identity:

$$
\sum_{v} \text { evweight } v=\frac{M(0, r+1)}{1-\sum_{0 \leq k<l \leq r} U(l) s^{2 \chi(l \text { even })} X_{l} X_{k} M(k, l)},
$$

where the sum is over all words whose letters belong to the interval $[0, r]$.

Consider the homomorphism $\phi$ generated by:

$$
\phi\left(X_{k}\right):=u q^{k}(s Z)^{\chi(k \text { odd })} \quad(0 \leq k \leq r) .
$$

If $v=y_{1} y_{2} \cdots y_{n}$, then $\phi($ evweight $v)=u^{\lambda v} q^{\operatorname{tot} v} s^{2 \operatorname{evdec} v+\operatorname{odd} v} Z^{\text {odd } v}$. 


\section{A SEXTUPLE DISTRIBUTION}

Also

$$
\begin{aligned}
\phi(U(l)) & =\prod_{l \leq j \leq r}\left(1-u q^{j} s^{2 \chi(j \text { even })}(s Z)^{\chi(j \text { odd })}\right)^{-1} \\
\phi(M(k, l)) & =\prod_{k \leq j \leq l-1}\left(1-u q^{j}(s Z)^{\chi(j \text { odd })}\right)^{-1} .
\end{aligned}
$$

Now define

$$
\begin{aligned}
& (u)_{k}^{\prime}:= \begin{cases}1, & \text { if } k=0 \\
\prod_{0 \leq j \leq k-1}\left(1-u q^{j}(s Z)^{\chi(j \text { odd })}\right), & \text { if } k \geq 1\end{cases} \\
& (u)_{l}^{\prime \prime}:= \begin{cases}1, & \text { if } l=0 \\
\prod_{1 \leq j \leq l}\left(1-u q^{j} s^{2 \chi(j \text { even })}(s Z)^{\chi(j \text { odd })}\right), & \text { if } l \geq 1 .\end{cases}
\end{aligned}
$$

Then $\phi(U(l))=\frac{(u)_{l-1}^{\prime \prime}}{(u)_{r}^{\prime \prime}} \quad(1 \leq l) ; \quad \phi(M(k, l))=\frac{(u)_{k}^{\prime}}{(u)_{l}^{\prime}} \quad(0 \leq k<l)$.

Now, take the image of the identity of Theorem 6.2 under $\phi$ :

$$
\sum_{w \in[0, r]^{*}} u^{\lambda w} q^{\text {tot } w} s^{(2 \text { evdec }+ \text { odd }) w} Z^{\text {odd } w}=\sum_{w \in[0, r]^{*}} \phi(\text { evweight } w)=\frac{1}{(u)_{r+1}^{\prime}} \frac{1}{S},
$$

where

$$
S=1-\sum_{0 \leq k<l \leq r} \frac{(u)_{l-1}^{\prime \prime}}{(u)_{r}^{\prime \prime}} s^{2 \chi(l \text { even })} u q^{l}(s Z)^{\chi(l \text { odd })} u q^{k}(s Z)^{\chi(k \text { odd })} \frac{(u)_{k}^{\prime}}{(u)_{l}^{\prime}} .
$$

As $-u q^{k}(s Z)^{\chi(k \text { odd })}(u)_{k}^{\prime}=(u)_{k+1}^{\prime}-(u)_{k}^{\prime}$, we have:

$$
S=1+\sum_{1 \leq l \leq r} \frac{(u)_{l-1}^{\prime \prime}}{(u)_{r}^{\prime \prime}} s^{2 \chi(l \text { even })} u q^{l}(s Z)^{\chi(l \text { odd })} \frac{1}{(u)_{l}^{\prime}}\left((u)_{l}^{\prime}-1\right)
$$

Now, $-u q^{l} s^{2 \chi(l \text { even })}(s Z)^{\chi(l \text { odd })}(u)_{l-1}^{\prime \prime}=(u)_{l}^{\prime \prime}-(u)_{l-1}^{\prime \prime}$. Hence,

$$
S=\frac{1}{(u)_{r}^{\prime \prime}}-\frac{1}{(u)_{r}^{\prime \prime}} \sum_{1 \leq l \leq r} \frac{(u)_{l-1}^{\prime \prime}}{(u)_{l}^{\prime}} u q^{l} s^{2 \chi(l \text { even })}(s Z)^{\chi(l \text { odd })}
$$

We are then left to prove:

$$
\frac{(u)_{r}^{\prime \prime}}{(u)_{r+1}^{\prime}}\left(1-\sum_{1 \leq l \leq r} \frac{(u)_{l-1}^{\prime \prime}}{(u)_{l}^{\prime}} u q^{l} s^{2 \chi(l \text { even })}(s Z)^{\chi(l \text { odd })}\right)^{-1}=F_{r}(u ; s, q, Z)
$$


We can also write:

$$
\begin{gathered}
(u)_{r}^{\prime}=\left(u ; q^{2}\right)_{\lfloor(r+1) / 2\rfloor}\left(u s q Z ; q^{2}\right)_{\lfloor r / 2\rfloor}, \\
(u)_{r}^{\prime \prime}=\left(u s q Z ; q^{2}\right)_{\lfloor(r+1) / 2\rfloor}\left(u s^{2} q^{2} ; q^{2}\right)_{\lfloor r / 2\rfloor},
\end{gathered}
$$

so that

$$
\frac{(u)_{r}^{\prime \prime}}{(u)_{r+1}^{\prime}}=\frac{\left(u s^{2} q^{2} ; q^{2}\right)_{\lfloor r / 2\rfloor}}{\left(u ; q^{2}\right)_{\lfloor r / 2\rfloor+1}} \quad \text { and } \quad \frac{(u)_{l-1}^{\prime \prime}}{(u)_{l}^{\prime}}=\frac{\left(u s^{2} q^{2} ; q^{2}\right)_{\lfloor(l-1) / 2\rfloor}}{\left(u ; q^{2}\right)_{\lfloor(l+1) / 2\rfloor}}
$$

Identity (6.2) may be rewritten as

$$
\frac{\left(u s^{2} q^{2} ; q^{2}\right)_{\lfloor r / 2\rfloor}}{\left(u ; q^{2}\right)_{\lfloor r / 2\rfloor+1}}(1-T)^{-1}=F_{r}(u ; s, q, Z),
$$

where

$$
\begin{aligned}
T & :=\sum_{1 \leq l \leq r} \frac{\left(u s^{2} q^{2} ; q^{2}\right)_{\lfloor(l-1) / 2\rfloor}}{\left(u ; q^{2}\right)_{\lfloor(l+1) / 2\rfloor}} u q^{l} s^{2 \chi(l \text { even })}(s Z)^{\chi(l \text { odd })} \\
& =\sum_{0 \leq l \leq\lfloor(r-1) / 2\rfloor} \frac{\left(u s^{2} q^{2} ; q^{2}\right)_{l}}{\left(u ; q^{2}\right)_{l+1}} u q^{2 l} s q Z+\sum_{0 \leq l \leq\lfloor r / 2\rfloor-1} \frac{\left(u s^{2} q^{2} ; q^{2}\right)_{l}}{\left(u ; q^{2}\right)_{l+1}} u q^{2 l} s^{2} q^{2} .
\end{aligned}
$$

We can then introduce

$$
G(m):=\sum_{0 \leq l \leq m} \frac{\left(u s^{2} q^{2} ; q^{2}\right)_{l}}{\left(u ; q^{2}\right)_{l+1}} u q^{2 l}
$$

and try to sum it. As

$$
\frac{\left(u s^{2} q^{2} ; q^{2}\right)_{l+1}}{\left(u ; q^{2}\right)_{l+1}}-\frac{\left(u s^{2} q^{2} ; q^{2}\right)_{l}}{\left(u ; q^{2}\right)_{l}}=\frac{\left(u s^{2} q^{2} ; q^{2}\right)_{l}}{\left(u ; q^{2}\right)_{l+1}} u q^{2 l}\left(1-s^{2} q^{2}\right),
$$

we get

$$
G(m)=\frac{1}{1-s^{2} q^{2}}\left(\frac{\left(u s^{2} q^{2} ; q^{2}\right)_{m+1}}{\left(u ; q^{2}\right)_{m+1}}-1\right)
$$

so that

$$
\begin{aligned}
& T=s q Z G(\lfloor(r-1) / 2\rfloor)+s^{2} q^{2} G(\lfloor r / 2\rfloor-1) \\
& =\frac{1}{1-s^{2} q^{2}}\left(s q Z \frac{\left(u s^{2} q^{2} ; q^{2}\right)\lfloor(r+1) / 2\rfloor}{\left(u ; q^{2}\right)\lfloor(r+1) / 2\rfloor}-s q Z\right. \\
& \left.\quad+s^{2} q^{2} \frac{\left(u s^{2} q^{2} ; q^{2}\right)\lfloor r / 2\rfloor}{\left(u ; q^{2}\right)\lfloor r / 2\rfloor}-s^{2} q^{2}\right) .
\end{aligned}
$$

By reporting this value of $T$ into (6.4) we exactly find the expression of $F_{r}(u ; s, q, Z)$ displayed in (1.5). 


\section{A SEXTUPLE DISTRIBUTION}

\section{Second proof of (5.1)}

When $Z=0$ in $B_{n}\left(s, t, q, Y_{0}, Y_{1}, Z\right)$, then $Y_{1}$ is also null. Furthermore, each polynomial $B_{n}\left(s, t, q, Y_{0}, 0,0\right)$ is a polynomial in $s^{2}, t^{2}, q^{2}, Y_{0}$. The summation on the right-hand side of (1.6) only involves even powers of $t$. We then have

$$
F_{2 r}(u ; s, q, 0)=\frac{\left(u s^{2} q^{2} ; q^{2}\right)_{r}\left(1-s^{2} q^{2}\right)\left(u ; q^{2}\right)_{r}}{\left(u ; q^{2}\right)_{r+1}\left(\left(u ; q^{2}\right)_{r}-s^{2} q^{2}\left(u s^{2} q^{2} ; q^{2}\right)_{r}\right)}
$$

and

$$
\sum_{n \geq 0} B_{n}\left(s, t, q, Y_{0}, 0,0\right) \frac{u^{n}}{\left(t^{2} ; q^{2}\right)_{n+1}}=\sum_{r \geq 0} t^{2 r} \frac{\left(u ; q^{2}\right)_{r+1}}{\left(u Y_{0} ; q^{2}\right)_{r+1}} F_{2 r}(u ; s, q, 0) .
$$

But $B_{n}\left(s^{1 / 2}, t^{1 / 2}, q^{1 / 2}, Y, 0,0\right)$ is the generating polynomial $A_{n}(s, t, q, Y)$ for the symmetric group $\mathfrak{S}_{n}$ by the statistic (exc, des, maj, fix) and it was proved in our previous paper [FoHa06b] that

$\sum_{n \geq 0} A_{n}(s, t, q, Y) \frac{u^{n}}{(t ; q)_{n+1}}=\sum_{r \geq 0} t^{r} \frac{(u ; q)_{r+1}}{(u Y ; q)_{r+1}} \frac{(u s q ; q)_{r}(1-s q)(u ; q)_{r}}{(u ; q)_{r+1}\left((u ; q)_{r}-s q(u s q ; q)_{r}\right)}$

Accordingly, (1.4) holds when $Z=0$ and consequently (5.1) holds when $r$ is even and $Z$ null. To show that identity (5.1), which we shall rewrite as

$$
\sum_{v \in[0, r]^{*}} \operatorname{EV}(v)=F_{r}(u ; s, q, Z)
$$

is true for all values of $r$ and when $Z$ is not necessarily 0 we proceed as follows.

Cut each word $v \in[0, r]^{*}$ in the (5.1) summation after every occurrence of an odd letter. This defines a unique factorization:

$$
v=p_{0} i_{0} p_{1} i_{1} \cdots p_{k} i_{k} p_{k+1}
$$

of $v$ having the following properties

(C1) $k \geq 0$,

(C2) $p_{0}, p_{1}, \ldots, p_{k}, p_{k+1}$ all from $P_{r}^{*}$ with $P_{r}:=\{0,2,4, \ldots, 2\lfloor r / 2\rfloor\}$,

(C3) $i_{0}, i_{1}, \ldots, i_{k}$ all from $I_{r}$ with $I_{r}:=\{1,3,5, \ldots, 2\lfloor(r+1) / 2\rfloor-1\}$.

The following properties of this factorization are easy to verify:

$(\mathrm{P} 1) \operatorname{tot} v=\operatorname{tot}\left(p_{0} i_{0}\right)+\operatorname{tot}\left(p_{1} i_{1}\right)+\cdots+\operatorname{tot}\left(p_{k} i_{k}\right)+\operatorname{tot}\left(p_{k+1}\right)$,

$(\mathrm{P} 2) \operatorname{evdec} v=\operatorname{evdec}\left(p_{0} i_{0}\right)+\operatorname{evdec}\left(p_{1} i_{1}\right)+\cdots+\operatorname{evdec}\left(p_{k} i_{k}\right)+\operatorname{evdec}\left(p_{k+1}\right)$,

(P3) odd $v=\operatorname{odd}\left(p_{0} i_{0}\right)+\operatorname{odd}\left(p_{1} i_{1}\right)+\cdots+\operatorname{odd}\left(p_{k} i_{k}\right)+\operatorname{odd}\left(p_{k+1}\right)$,

(P4) $\lambda v=\lambda\left(p_{0} i_{0}\right)+\lambda\left(p_{1} i_{1}\right)+\cdots+\lambda\left(p_{k} i_{k}\right)+\lambda\left(p_{k+1}\right)$, 
(P5) $\mathrm{EV}\left(p_{0} i_{0} p_{1} i_{1} \cdots p_{k} i_{k} p_{k+1}\right)=\mathrm{EV}\left(p_{0} i_{0}\right) \mathrm{EV}\left(p_{1} i_{1}\right) \cdots \mathrm{EV}\left(p_{k} i_{k}\right) \operatorname{EV}\left(p_{k+1}\right)$,

(P6) $\mathrm{EV}(p i)=s q Z \mathrm{EV}(p(i-1))$ for $p \in P_{r}^{*}$ and $i \in I_{r}$,

(P7) $\operatorname{EV}(p(2 k))=u q^{2 k} \operatorname{EV}(p)$ for $p \in P_{2 k}^{*}$.

Hence

$$
\begin{aligned}
\sum_{v \in[0, r]^{*}} v & =\left(1-\sum_{p \in P_{r}^{*}, i \in I_{r}} p i\right)^{-1} \sum_{p \in P_{r}^{*}} p \\
\sum_{v \in[0, r]^{*}} \operatorname{EV}(v) & =\frac{1}{1-\sum_{p \in P_{r}^{*}, i \in I_{r}} \operatorname{EV}(p i)} \sum_{p \in P_{r}^{*}} \operatorname{EV}(p) .
\end{aligned}
$$

On the other hand,

$$
\sum_{p \in P_{r}^{*}, i \in I_{r}} \operatorname{EV}(p i)=s q Z \sum_{p \in P_{r}^{*}, i \in I_{r}} \operatorname{EV}(p(i-1))=s q Z \sum_{p \in P_{r}^{*}, j \in P_{r-1}} \operatorname{EV}(p j) .
$$

If $r=2 k+1$, then $P_{r}=P_{r-1}=P_{2 k}$, so that

$$
\sum_{p \in P_{r}^{*}, j \in P_{r-1}} \operatorname{EV}(p j)=\sum_{p \in P_{2 k}^{*}, \lambda(p) \geq 1} \operatorname{EV}(p)=F_{2 k}(u ; s, q, 0)-1,
$$

because (7.3) holds for $r$ even and $Z=0$. It follows from (7.5) that

$$
\sum_{v \in[0,2 k+1]^{*}} \operatorname{EV}(v)=\frac{F_{2 k}(u ; s, q, 0)}{1-s q Z\left(F_{2 k}(u ; s, q, 0)-1\right)} .
$$

If $r=2 k$, then $P_{r}=P_{2 k}$ and $P_{r-1}=P_{2 k-2}$, so that

$$
\begin{aligned}
\sum_{p \in P_{r}^{*}, j \in P_{r-1}} \operatorname{EV}(p j) & =\sum_{p \in P_{2 k}^{*}, j \in P_{2 k-2}} \operatorname{EV}(p j) \\
& =\sum_{p \in P_{2 k}^{*}, \lambda(p) \geq 1} \operatorname{EV}(p)-\sum_{p \in P_{2 k}^{*}} \operatorname{EV}(p(2 k)) \\
& =\left(F_{2 k}(u ; s, q, 0)-1\right)-u q^{2 k} F_{2 k}(u ; s, q, 0) \\
& =\left(1-u q^{2 k}\right) F_{2 k}(u ; s, q, 0)-1 .
\end{aligned}
$$

Hence (7.5) implies

$$
\sum_{v \in[0,2 k]^{*}} \operatorname{EV}(v)=\frac{F_{2 k}(u ; s, q, 0)}{1-s q Z\left(\left(1-u q^{2 k}\right) F_{2 k}(u ; s, q, 0)-1\right)} .
$$

We can then report the value of $F_{2 k}(u ; s, q, 0)$ obtained in (7.1) in both expressions (7.6) and (7.7). By combining them in a single formula we exactly get the formula displayed in $(1.5)$ for $F_{r}(u ; s, q, Z)$. 


\section{A SEXTUPLE DISTRIBUTION}

\section{Two proofs of Corollary $\mathbf{1 . 3}$}

For the first proof we proceed as follows. Let $Z=1$ in (1.5) and write the formula as:

We have

$$
\begin{aligned}
& \frac{\left(u s^{2} q^{2} ; q^{2}\right)_{\lfloor r / 2\rfloor}\left(1-s^{2} q^{2}\right)\left(u ; q^{2}\right)_{\lfloor(r+1) / 2\rfloor}}{\left(u ; q^{2}\right)_{\lfloor r / 2\rfloor+1} F_{r}(u ; s, q, 1)} \\
& \quad=\left(u ; q^{2}\right)_{\lfloor(r+1) / 2\rfloor}-s^{2} q^{2} \frac{\left(u ; q^{2}\right)_{\lfloor(r+1) / 2\rfloor}}{\left(u ; q^{2}\right)_{\lfloor r / 2\rfloor}}\left(u s^{2} q^{2} ; q^{2}\right)\lfloor r / 2\rfloor \\
& \quad+s q\left(u ; q^{2}\right)\lfloor(r+1) / 2\rfloor-s q\left(u s^{2} q^{2} ; q^{2}\right)\lfloor(r+1) / 2\rfloor
\end{aligned}
$$

$$
\begin{aligned}
& s^{2} q^{2} \frac{\left(u ; q^{2}\right)_{\lfloor(r+1) / 2\rfloor}}{\left(u ; q^{2}\right)_{\lfloor r / 2\rfloor}}\left(u s^{2} q^{2} ; q^{2}\right)_{\lfloor r / 2\rfloor}+s q\left(u s^{2} q^{2} ; q^{2}\right)_{\lfloor(r+1) / 2\rfloor} \\
& =s q\left(u s^{2} q^{2} ; q^{2}\right)_{\lfloor r / 2\rfloor}\left(s q\left(1-u q^{2\lfloor(r-1) / 2\rfloor}\right)+1-u s^{2} q^{2\lfloor(r-1) / 2\rfloor+2}\right) \\
& =s q\left(u s^{2} q^{2} ; q^{2}\right)_{\lfloor r / 2\rfloor}(1+s q)\left(1-u s q^{2\lfloor(r-1) / 2\rfloor+1}\right) \\
& =s q\left(u s^{2} q^{2} ; q^{2}\right)_{\lfloor r / 2\rfloor}(1+s q) \frac{\left(u s q ; q^{2}\right)_{\lfloor(r+1) / 2\rfloor}}{\left(u s q ; q^{2}\right)_{\lfloor r / 2\rfloor}},
\end{aligned}
$$

so that

$$
\begin{aligned}
& \frac{\left(u s^{2} q^{2} ; q^{2}\right)_{\lfloor r / 2\rfloor}\left(1-s^{2} q^{2}\right)\left(u ; q^{2}\right)_{\lfloor(r+1) / 2\rfloor}}{\left(u ; q^{2}\right)_{\lfloor r / 2\rfloor+1} F_{r}(u ; s, q, 1)} \\
& \quad=(1+s q)\left(\left(u ; q^{2}\right)_{\lfloor(r+1) / 2\rfloor}-s q\left(u s^{2} q^{2} ; q^{2}\right)_{\lfloor r / 2\rfloor} \frac{\left(u s q ; q^{2}\right)_{\lfloor(r+1) / 2\rfloor}}{\left(u s q ; q^{2}\right)_{\lfloor r / 2\rfloor}} .\right.
\end{aligned}
$$

By dividing both sides by $(1+s q)$ we recover $(1.8)$.

For the second proof we again use the notations introduced in Section 7, namely,

$$
\begin{aligned}
(u)_{r}^{\prime} & := \begin{cases}1, & \text { if } r=0 ; \\
\prod_{0 \leq j \leq r-1}\left(1-u q^{j}(s Z)^{\chi(j \text { odd })}\right), & \text { if } r \geq 1 ;\end{cases} \\
& =\left(u ; q^{2}\right)_{\lfloor(r+1) / 2\rfloor}\left(u s q Z ; q^{2}\right)_{\lfloor r / 2\rfloor},
\end{aligned}
$$

and directly prove the identity

$$
\sum_{v \in[0, r]^{n}} q^{\operatorname{tot} v} s^{2 \text { evdec } v+\operatorname{odd} v}=F_{r}(u ; s, q, 1),
$$

where $F_{r}(u ; s, q, 1)$ is given by $(1.8)$, an identity that may be rewritten as:

$$
\sum_{v \in[0, r]^{n}} q^{\operatorname{tot} v} s^{2 \operatorname{evdec} v+\operatorname{odd} v}=\frac{1}{(u)_{r+1}^{\prime}} \times \frac{(1-s q)(u)_{r}^{\prime}(u s q)_{r}^{\prime}}{(u)_{r}^{\prime}-s q(u s q)_{r}^{\prime}} .
$$




\section{DOMINIQUE FOATA AND GUO-NIU HAN}

For each $r \geq 0$ let $D(r)$ be the set of all pairs $(w, i)$ such that $w \in \operatorname{NIW}(r-1), 1 \leq i \leq \lambda w-1, \lambda w \geq 2$. In our previous paper ([FoHa07b], Theorem 2.1) we have constructed a bijection mapping each word $v$ onto a sequence

$$
\left(w_{0},\left(w_{1}, i_{1}\right),\left(w_{2}, i_{2}\right), \ldots,\left(w_{k}, i_{k}\right)\right)
$$

such that $w_{0} \in \operatorname{NIW}(r)$ and each pair $\left(w_{l}, i_{l}\right) \in D(r)(1 \leq l \leq k)$ and $w_{0} w_{1} \cdots w_{k}$ is a rearrangement of $v$. Furthermore, the bijection has the following property:

(8.2) 2 evdec $v+\operatorname{odd} v=i_{1}+i_{2}+\cdots i_{k}+\operatorname{odd} w_{0}+\operatorname{odd} w_{1}+\cdots+\operatorname{odd} w_{k}$.

Hence, $\sum_{v \in[0, r]^{n}} q^{\operatorname{tot} v} s^{2 \operatorname{evdec} v+\operatorname{odd} v}=\frac{A}{1-B}$, where $A=\sum_{w \in \operatorname{NIW}(r)} q^{\operatorname{tot} w} s^{\text {odd } w} u^{\lambda w}$ and $B=\sum_{(w, i) \in D(r)}(s q)^{i} q^{\text {tot } w} s^{\text {odd } w} u^{\lambda w}$. But $A=\frac{1}{(u)_{r+1}^{\prime}}$ and

$$
\begin{aligned}
B= & \sum_{(w, i) \in D(r)}(s q)^{i} q^{\text {tot } w} s^{\text {odd } w} u^{\lambda w} \\
= & \sum_{n \geq 2} \sum_{i=1}^{n-1}(s q)^{i} \sum_{w \in \operatorname{NIW}_{n}(r-1)} q^{\operatorname{tot} w} s^{\text {odd } w} u^{\lambda w} \\
= & \sum_{n \geq 2} \frac{s q-(s q)^{n}}{1-s q} \sum_{w \in \operatorname{NIW}_{n}(r-1)} q^{\operatorname{tot} w} s^{\text {odd } w} u^{\lambda w} \\
= & \frac{s q}{1-s q} \sum_{n \geq 2} \sum_{w \in \mathrm{NIW}_{n}(r-1)} q^{\operatorname{tot} w} s^{\text {odd } w} u^{\lambda w} \\
& +\frac{1}{1-s q} \sum_{n \geq 2} \sum_{w \in \mathrm{NIW}_{n}(r-1)} q^{\operatorname{tot} w} s^{\text {odd } w}(u s q)^{\lambda w} \\
= & \frac{s q}{1-s q}\left(\frac{1}{(u)_{r}^{\prime}}-(1+c u)\right)+\frac{1}{1-s q}\left(\frac{1}{(u s q)_{r}^{\prime}}-(1+c u s q)\right)
\end{aligned}
$$

[where $c$ is the coefficient of $u$ in $1 /(u)_{r}^{\prime}$ ]

$$
=1+\frac{1}{1-s q}\left(\frac{s q}{(u)_{r}^{\prime}}-\frac{1}{(u s q)_{r}^{\prime}}\right) \text {. }
$$

Finally, $\sum_{v \in[0, r]^{n}} q^{\operatorname{tot} v} s^{2 \operatorname{evdec} v+\operatorname{odd} v}=\frac{A}{1-B}=\frac{1}{(u)_{r+1}^{\prime}} \times \frac{(1-s q)(u)_{r}^{\prime}(u s q)_{r}^{\prime}}{(u)_{r}^{\prime}-s q(u s q)_{r}^{\prime}}$, which is the expression written in (8.1).

Remark. The method used in the latter proof cannot be applied when $Z \neq 1$. Although identity (8.2) always holds, we do not have

$$
\operatorname{odd} v=\operatorname{odd} w_{0}+\operatorname{odd} w_{1}+\cdots+\operatorname{odd} w_{k}
$$

in general. 


\section{A SEXTUPLE DISTRIBUTION}

\section{Specializations}

In Section 7 we have seen that the generating function for the polynomials $A_{n}(s, t, q, Y)$ can be derived from (1.6) by letting $Z=0$ and replace the triplet $\left(s, t, q, Y_{0}\right)$ by $\left(s^{1 / 2}, t^{1 / 2}, q^{1 / 2}, Y\right)$. We just comment the specializations $s=1$ and $Y_{0}=Y_{1}=Z=1$.

9.1. Case $s=1$. We have

$$
\begin{gathered}
F_{r}(u ; 1, q, Z) \\
=\frac{\left(u q^{2} ; q^{2}\right)_{\lfloor r / 2\rfloor}\left(1-q^{2}\right)\left(u ; q^{2}\right)_{\lfloor(r+1) / 2\rfloor}\left(u ; q^{2}\right)_{\lfloor r / 2\rfloor}}{\left(u ; q^{2}\right)_{\lfloor r / 2\rfloor+1}\left(\left(u ; q^{2}\right)_{\lfloor(r+1) / 2\rfloor}\left(\left(u ; q^{2}\right)_{\lfloor r / 2\rfloor}-q^{2}\left(u q^{2} ; q^{2}\right)_{\lfloor r / 2\rfloor}\right)\right.} \\
\left.+q Z\left(u ; q^{2}\right)_{\lfloor r / 2\rfloor}\left(\left(u ; q^{2}\right)_{\lfloor(r+1) / 2\rfloor}-\left(u q^{2} ; q^{2}\right)_{\lfloor(r+1) / 2\rfloor}\right)\right),
\end{gathered}
$$

so that we can divide both numerator and denominator by the product $\left(u ; q^{2}\right)_{\lfloor(r+1) / 2\rfloor}\left(u ; q^{2}\right)_{\lfloor r / 2\rfloor}$. We then get

$$
\begin{aligned}
F_{r}(u ; 1, q, Z) & =\frac{1-q^{2}}{1-u}\left(1-q^{2} \frac{1-u q^{2\lfloor r / 2\rfloor}}{1-u}+q Z-q Z \frac{1-u q^{2\lfloor(r+1) / 2\rfloor}}{1-u}\right)^{-1} \\
& =\left(1-u \frac{1-q^{2\lfloor r / 2\rfloor+2}}{1-q^{2}}-u q Z \frac{1-q^{2\lfloor(r+1) / 2\rfloor}}{1-q^{2}}\right)^{-1} \\
& =\left(1-u \sum_{0 \leq i \leq r} q^{i} Z^{\chi(i \text { odd })}\right)^{-1} .
\end{aligned}
$$

We then recover identity (1.9) from our paper [FoHa07a] in the form:

$$
\begin{aligned}
& \text { 9.1) } \sum_{n \geq 0}(1+t) B_{n}\left(1, t, q, Y_{0}, Y_{1}, Z\right) \frac{u^{n}}{\left(t^{2} ; q^{2}\right)_{n+1}} \\
& =\sum_{r \geq 0} t^{r} \frac{\left(u ; q^{2}\right)_{\lfloor r / 2\rfloor+1}}{\left(u Y_{0} ; q^{2}\right)_{\lfloor r / 2\rfloor+1}} \frac{\left(-u q Y_{1} Z ; q^{2}\right)_{\lfloor(r+1) / 2\rfloor}}{\left(-u q Z ; q^{2}\right)_{\lfloor(r+1) / 2\rfloor}}\left(1-u \sum_{i=0}^{r} q^{i} Z^{\chi(i \text { odd })}\right)^{-1} .
\end{aligned}
$$

9.2. Case $Y_{0}=Y_{1}=Z=1$. Identity (1.7) simply becomes

$$
\sum_{n \geq 0}(1+t) B_{n}(s, t, q, 1,1,1) \frac{u^{n}}{\left(t^{2} ; q^{2}\right)_{n+1}}=\sum_{r \geq 0} t^{r} F_{r}(u ; s, q, 1),
$$

where $F_{r}(u ; s, q, 1)$ is given by (1.8). Note that $B_{n}(s, t, q, 1,1,1)$ is the generating polynomial for the group $B_{n}$ by (fexc, fdes, fmaj). Write $B_{n}(s, t)$ for $B_{n}(s, t, 1,1,1,1)$. From (9.2) we deduce

$$
\begin{aligned}
\sum_{n \geq 0} B_{n}(s, t) \frac{u^{n}}{\left(1-t^{2}\right)^{n}}=\sum_{r \geq 0} & \left(\frac{(1-s)(1-t) t^{2 r}}{(1-u)^{r+1}\left(1-u s^{2}\right)^{-r}-s(1-u)}\right. \\
& \left.+\frac{(1-s)(1-t) t^{2 r+1}}{(1-u)^{r+1}\left(1-u s^{2}\right)^{-r}-s(1-u s)}\right)
\end{aligned}
$$


To obtain the marginal distribution $B_{n}(s, 1)=\sum_{w \in B_{n}} s^{\text {fexc } w}$ it is convenient
to specialize formula (1.9). We obtain

$$
\sum_{n \geq 0} B_{n}(s, 1) \frac{u^{n}}{n !}=\frac{1-s}{-s+\exp \left(u\left(s^{2}-1\right)\right)} .
$$

As for $B_{n}(1, t)=\sum_{w \in B_{n}} t^{\mathrm{fdes} w}$ we specialize (9.1) and find:

$$
\sum_{n \geq 0} B_{n}(1, t) \frac{u^{n}}{\left(1-t^{2}\right)^{n}}=\sum_{r \geq 0} t^{r} \frac{1-t}{1-u(r+1)} .
$$

Hence, $B_{n}(1, t) /\left(1-t^{2}\right)^{n}=(1-t) \sum_{r \geq 0} t^{r}(r+1)^{n}$ and

$$
\sum_{n \geq 0} B_{n}(1, t) \frac{u^{n}}{n !}=\frac{1-t}{-t+\exp \left(u\left(t^{2}-1\right)\right)} .
$$

It follows from (9.4) and (9.6) that $B_{n}(s, 1)=B_{n}(1, s)$, so that "fexc" and "fdes" are equally distributed on the group $B_{n}$. For $k, n \geq 0$ let $B_{n, k}$ be the number of signed permutations $w$ from $B_{n}$ such that fdes $w=k$. In our paper [FoHa06] we have derived the recurrence formula for the $B_{n, k}$ 's in a more general context. The recurrence reads as follows

$$
\begin{gathered}
B_{0,0}=1, \quad B_{0, k}=0 \text { for all } k \neq 0 \\
B_{1,0}=1, \quad B_{1,1}=1, \quad B_{1, k}=0 \text { for all } k \neq 0,1 ; \\
B_{n, k}=(k+1) B_{n-1, k}+B_{n-1, k-1}+(2 n-k) B_{n-1, k-2}
\end{gathered}
$$

for $n \geq 2$ and $0 \leq k \leq 2 n-1$. Let $B_{n, k}^{\prime}:=\#\left\{w \in B_{n}\right.$ : fexc $\left.w=k\right\}$. The coefficients $B_{n, k}^{\prime}$ 's satisfy the same recurrence as the $B_{n, k}$ 's. The induction is easy, so that we can fabricate a bijection $\psi$ of $B_{n}$ onto itself such that fexc $w=\operatorname{fdes} \psi(w)$.

\section{Concluding remarks}

The statistical study of the group $B_{n}$ and some other Weyl groups has been initiated by Reiner ([Re93a], [Re93b], [Re93c], [Re95a], [Re95b]) and continued by the Roman school ([Br94], [Bi03], [BiCa04]). It has been rejuvenated by Adin, Roichman [AR01] with their definition of the flag major index for signed permutations and the first proof of the fact that length function and flag-major index were equidistributed over $B_{n}$ ([ABR01], [ABR05], [ABR06]). In our series "Signed words and permutations; I-V" we have tried to work out analytical expressions for the multivariable distributions on the group $B_{n}$ that were natural extensions of 


\section{A SEXTUPLE DISTRIBUTION}

the expressions already derived for the symmetric group $\mathfrak{S}_{n}$. Other works along those lines are due to Gessel and his school ([ChGe07], [Ch03]). Further algebraic extensions have recently been done by the Minnesota school [BRS07].

We should like to thank Christian Krattenthaler [Kr07] who urged us to use the telescoping technique to shorten our $q$-calculations (see Section 6).

\section{References}

[AR01] Ron M. Adin and Yuval Roichman. The flag major index and group actions on polynomial rings, Europ. J. Combin., vol. 22, 2001, p. 431-446.

[ABR01] Ron M. Adin, Francesco Brenti and Yuval Roichman. Descent Numbers and Major Indices for the Hyperoctahedral Group, Adv. in Appl. Math., vol. 27, 2001, p. 210-224.

[ABR05] Ron M. Adin, Francesco Brenti and Yuval Roichman. Descent representations and multivariate statistics, Trans. Amer. Math. Soc., vol. 357, 2005, p. 30513082 .

[ABR06] Ron M. Adin, Francesco Brenti and Yuval Roichman. Equi-distribution over Descent Classes of the Hyperoctahedral Group, J. Comb. Theory, Ser. A., vol. 113, 2006, p. 917-933.

[An76] George E. Andrews. The Theory of Partitions. Addison-Wesley, Reading MA, 1976 (Encyclopedia of Math.and its Appl., 2).

[BRS07] Hélène Barcelo, Victor Reiner, Dennis Stanton. Bimahonian Distributions, arXiv:math.CO/0703479v1, 16 Mar 2007, 21 pages.

[Bi03] Riccardo Biagioli. Major and descent statistics for the even-signed permutation group, Adv. in Appl. Math., vol. 31, 2003, p. 163-179.

[BiCa04] Riccardo Biagioli and Fabrizio Caselli. Invariant algebras and major indices for classical Weyl groups, Proc. London Math. Soc., vol. 88, 2004, p. 603-631.

[Br94] Francesco Brenti. $q$-Eulerian Polynomials Arising from Coxeter Groups, Europ. J. Combinatorics, vol. 15, 1994, p. 417-441.

[Bo68] N. Bourbaki. Groupes et algèbres de Lie, chap. 4, 5, 6. Hermann, Paris, 1968.

[Ch03] Chak-On-Chow. On the Eulerian polynomials of type D, Europ. J. Combin, vol. 24, 2003, p. 391-408.

[ChGe07] Chak-On-Chow, Ira M. Gessel. On the descent numbers and major indices for the hyuperoctahedral group, Adv. in Appl. Math., vol. 38, 2007, p. 275-301.

[Ch58] K.T. Chen, R.H. Fox, R.C. Lyndon. Free differential calculus, IV. The quotient group of the lower central series, Ann. of Math., vol. 68, 1958, p. 81-95.

[FoHa07] Dominique Foata and Guo-Niu Han. Signed Words and Permutations, I; a Fundamental Transformation, Proc. Amer. Math. Soc., vol. 135, 2006, p. 31-40.

[FoHa05] Dominique Foata and Guo-Niu Han. Signed Words and Permutations, II; The Euler-Mahonian Polynomials, Electronic J. Combinatorics (The Stanley Festschrift), vol. 11 (2), \#R22, 2004-2005,18 pages.

[FoHa06] Dominique Foata and Guo-Niu Han. Signed Words and Permutations, III; the MacMahon Verfahren, Sém. Lothar. Combin., 54 \#B54a (The Viennot Festschrift), 2005, 18 pages.

[FoHa07a] Dominique Foata and Guo-Niu Han. Signed Words and Permutations, IV; fixed and pixed points, to appear in Israel J. Math., 2007, 21 pages.

[FoHa07b] Dominique Foata and Guo-Niu Han. Fix-Mahonian Calculus, III; a quadruple distribution, to appear in Monatshefte für Math., 2007, 26 pages.

[GaRa90] George Gasper and Mizan Rahman. Basic Hypergeometric Series. London, Cambridge Univ. Press, 1990 (Encyclopedia of Math. and Its Appl., 35). 


\section{DOMINIQUE FOATA AND GUO-NIU HAN}

[GeRe93] Ira M. Gessel, Christophe Reutenauer. Counting Permutations with Given Cycle Structure and Descent Set, J. Combin. Theory, Ser. A, vol. 64, 1993, p. 189-215.

[Hu90] James E. Humphreys. Reflection Groups and Coxeter Groups. Cambridge Univ. Press, Cambridge (Cambridge Studies in Adv. Math., 29), 1990.

[KiZe01] Dongsu Kim, Jiang Zeng. A new decomposition of derangements, J. Combin. Theory Ser. A, vol. 96, 2001, p. 192-198.

[Kr07] Christian Krattenthaler. Private communication, 2007.

[Lo83] M. Lothaire. Combinatorics on Words. Addison-Wesley, London 1983 (Encyclopedia of Math. and its Appl., 17).

[Re93a] V. Reiner. Signed permutation statistics, Europ. J. Combinatorics, vol. 14, 1993, p. $553-567$.

[Re93b] V. Reiner. Signed permutation statistics and cycle type, Europ. J. Combinatorics, vol. 14, 1993, p. 569-579.

[Re93c] V. Reiner. Upper binomial posets and signed permutation statistics, Europ. J. Combinatorics, vol. 14, 1993, p. 581-588.

[Re95a] V. Reiner. Descents and one-dimensional characters for classical Weyl groups, Discrete Math., vol. 140, 1995, p. 129-140.

[Re95b] V. Reiner. The distribution of descents and length in a Coxeter group, Electronic J. Combinatorics, vol. 2, 1995, \# R25.

[Sch65] M.-P. Schützenberger. On a factorization of free monoids, Proc. Amer. Math. Soc., vol. 16, 1965, p. 21-24.

[ShWa06] John Shareshian, Michelle L. Wachs. $q$-Eulerian polynomials: excedance number and major index, Electronic Research Announcements of the Amer. Math. Soc., vol. 13, 2007, p. 33-45.

Dominique Foata

Institut Lothaire

1, rue Murner

F-67000 Strasbourg, France

foata@math.u-strasbg.fr
Guo-Niu Han

I.R.M.A. UMR 7501

Université Louis Pasteur et CNRS

7, rue René-Descartes

F-67084 Strasbourg, France

guoniu@math.u-strasbg.fr 